\title{
Measurement of the Elastic, Total and Single Diffraction Cross Sections at Tevatron Energies
}

\author{
Stefano Belforte \\ For the CDF Collaboration \\ INFN \\ Pisa, Italy \\ Fermi National Accelerator Laboratory \\ P.O. Box 500, Batavia, Illinois 60510
}

November 1993

To be published in the Proceedings of the Hadron '93 Conference, Como, Italy, June 21-25, 1993 


\section{Disclaimer}

This report was prepared as an account of work sponsored by an agency of the United States Government. Neither the United States Government nor any agency thereof, nor any of their employees, makes any warranty, express or implied, or assumes any legal liability or responsibility for the accuracy, completeness, or usefulness of any information, apparatus, product, or process disclosed, or represents that its use would not infringe privately owned rights. Reference herein to any specific commercial product, process, or service by trade name, trademark, manufacturer, or otherwise, does not necessarily constitute or imply its endorsement, recommendation, or favoring by the United States Government or any agency thereof. The views and opinions of authors expressed herein do not necessarily state or reflect those of the United States Government or any agency thereof. 


\title{
Measurement of the Elastic, Total and Single Diffraction Cross Sections at Tevatron Energies
}

\author{
Stefano Belforte \\ INFN, Pisa - Italy \\ for the CDF Collaboration
}

\begin{abstract}
CDF collaboration has measured the differential elastic cross section $d \sigma_{e l} / d t$, the single diffraction dissociation double differential cross section $d^{2} \sigma_{s d} / d M^{2} d t$ and the total inelastic cross section in antiprotonproton collisions at center of mass energies $\sqrt{s}=546$ and $1800 \mathrm{GeV}$. The elastic scattering slope is $15.28 \pm 0.58(16.98 \pm 0.25) \mathrm{GeV}^{-2}$ at $\sqrt{s}=546$ (1800) GeV. Using the luminosity independent method, $\left(1+\rho^{2}\right) \sigma_{T}$ is measured to be $62.64 \pm 0.95(81.83 \pm 2.29) \mathrm{mb}$ at $\sqrt{s}=546(1800)$ GeV. Assuming $\rho=0.15$, the elastic, total and single diffraction cross sections are $\sigma_{e l}=12.87 \pm 0.30, \sigma_{T}=61.26 \pm 0.93$ and $\sigma_{s d}=7.89 \pm 0.33$ $\mathrm{mb}\left(\sigma_{e l}=19.70 \pm 0.85, \sigma_{T}=80.03 \pm 2.24\right.$ and $\left.\sigma_{s d}=9.46 \pm 0.44 \mathrm{mb}\right)$ at 546 (1800) GeV.
\end{abstract}

PACS numbers: $13.85 . \mathrm{Dz}, 13.85 . \mathrm{Fb} .13 .85 . \mathrm{Hd}, 13.85 . \mathrm{Lg}$

\section{Experimental Method}

The luminosity-independent method is based on the simultaneous measurement of $d R_{e l} / d t$, the elastic scattering rate as a function of the four-momentum transfer-squared $(t)$, and of the total inelastic rate $R_{i n}$. The total cross section is then derived as $\sigma_{T}=\frac{16 \pi(\hbar c)^{2}}{1+\rho^{2}} \cdot \frac{d R_{e l} / d t t_{t=0}}{R_{e l}+R_{i n}}$ where $\rho$ is the ratio of the real

"To be published in the "Proceedings of the Hadron'93 Conference". June 21-25, 1993. Como - Italy 
to imaginary part of the forward elastic scattering amplitude. Data for the total cross section measurement have been collected in short dedicated runs during the 1988-1989 data taking period of CDF. Elastic scattering data have been collected in the four-momentum transfer range $0.025<-t<0.25 \mathrm{GeV}^{2}$. Single diffractive dissociation have been measured in the kinematical range $M_{x}^{2} / s<0.2$ and $0 \leq-t \leq 0.4 \mathrm{GeV}^{2}$.

The CDF detector has been described in detail in Ref. [1]. For these special runs, the CDF tracking system was supplemented with a small angle magnetic spectrometer in the accelerator tunnel and forward telescopes close to the detector.

The small angle spectrometer consisted of detectors placed between accelerator quadrupoles and dipoles that provided the bending fields. The detectors were arranged in two arms containing five movable beam pipe sections (roman pots) each, two measuring the outgoing proton trajectory (East side), and three measuring the outgoing antiproton trajectory (West side), placed at distance from the interaction point ranging from $z \simeq 30$ to $z \simeq 55 \mathrm{~m}$. Each roman pot contained a silicon detector and a drift chamber, sandwiched by two trigger counters. Both the silicon and the chamber measured both coordinates in the plane transverse to the beam direction at distances as close as $4 \mathrm{~mm}$ to the beam. The redundancy of detectors guaranteed full efficiency and reduces systematic uncertaineties.

The CDF detector acceptance for inelastic events was extended to $|\eta| \leq 6.7$ by the addition on each side of the interaction region of one telescope with 8 planes of tracking chambers followed by two roman pots at about $z=4 \mathrm{~m}$ containing tracking chambers and trigger counters. These counters, combined with the CDF Beam Beam Counters, provided a trigger signal on each side of the interaction region: East and West. On each side the trigger covered the pseudorapidity interval $3.2 \leq|\eta| \leq 6.7$.

A complete description of the apparatus and of the data analysis can be found in [2]. Results presented in the following are introduced by a short summary of the analysis procedure.

\section{Elastic Scattering}

Elastic events were triggered by the ten-fold coincidence of the counters in either spectrometer arm. A total of 34522 (38759) elastic triggers were collected at $546(1800) \mathrm{GeV}$. A preliminary selection removed triggers due to satellite bunches and high multiplicity events due to beam losses and interactions in the beam pipe. Space points were then reconstructed in each 

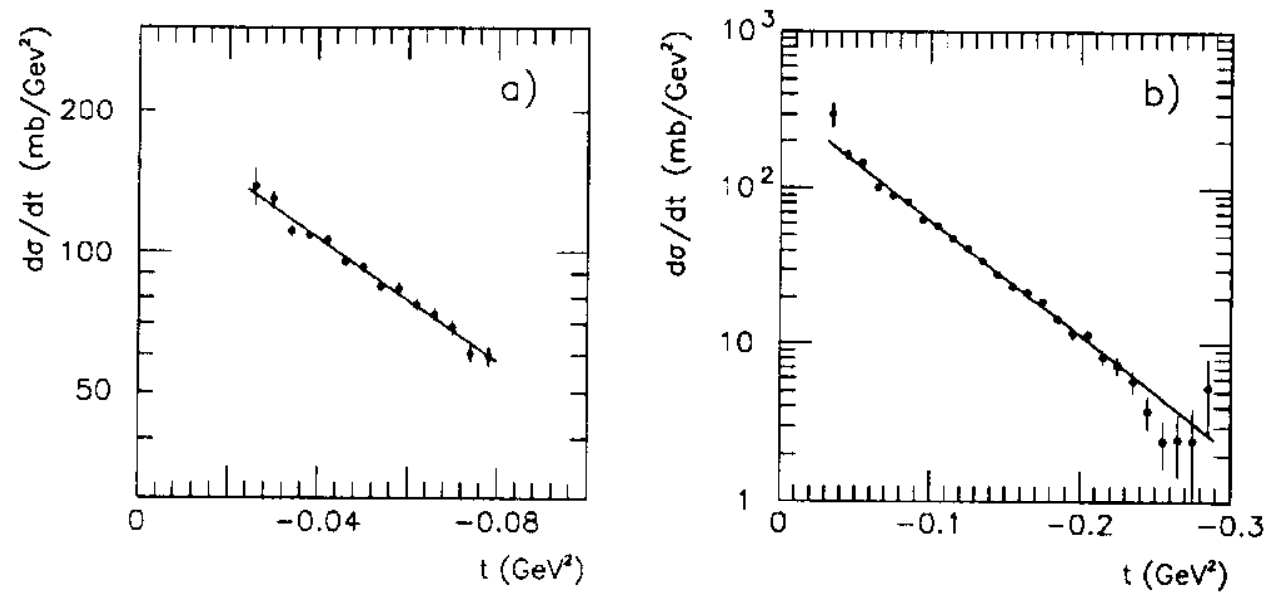

Figure 1: Differential cross section of proton-antiproton elastic scattering at (a) $\sqrt{s}=546 \mathrm{GeV}$ and at (b) $\sqrt{s}=1800 \mathrm{GeV}$. Lines represent the fit results described in the text.

pot and combined to identify the scattered proton and antiproton trajectory. Multiple tracks ambiguities have been solved by choosing the track pair with the best antiproton-proton collinearity. The final sample was obtained with a $3.5 \sigma$ cut on the distribution of the $\bar{p}$ trajectory impact parameter, followed by a $4 \sigma$ cut on the $\bar{p} p$ collinearity distributions. This sample contained 18919 (8744) events at $\sqrt{s}=546(1800) \mathrm{GeV}$. The residual background contamination $(\leq 0.5 \%)$ was estimated from the tails of the collinearity distribution and was statistically subtracted from the angular distribution. Corrections were also applied for losses due to the analysis cuts and to nuclear interactions in the detectors. The measured $d R_{e l} / d t$ distribution was then corrected for the spectrometer acceptance (obtained from a full Monte Carlo simulation of the detector). The elastic slope $b_{e l}$ and the optical point $A=d R_{e l} /\left.d t\right|_{t=0}$ were derived by fitting to the data the exponential form $A \cdot e^{b_{e l} t}$. Coulomb scattering corrections were taken into account, as well as the small smearing-effect of the detector resolution and of the beam width and divergency at the interaction point. The fit, shown in Fig. 1, yielded $b_{e l}=15.28 \pm 0.58(16.98 \pm 0.24) \mathrm{GeV}^{-2}$ at $546(1800) \mathrm{GeV}$. In order to obtain the optical point and the total number of elastic events, data at $\sqrt{s}=546 \mathrm{GeV}$ have been refitted making use of the more accurate values of the slope from the UA4 and UA4/2 [3] experiments: $b_{e l}=15.35 \pm 0.20 \mathrm{GeV}^{-2}$. The statistical error on A was $1.2 \%(3.0 \%)$ at 546 (1800) $\mathrm{GeV}$. The various sources of systematical errors on $b_{e l}$ and $A$ (uncer- 
tainties in event selection, background subtraction, transport in the magnetic fields, detectors alignment, $t$-scale and offset, correction for nuclear interactions) were carefully studied with the data and Monte Carlo simulations; they contributed a total additional $0.5 \%$ uncertainty on $A$ and $0.3 \%$ on $b_{e l}$ at both energies. Assuming an $s$-dependence of the slope $b_{e l}=b_{0}^{\prime}+2 \alpha^{\prime} \ln \left(s / s_{0}\right)$, the data at $\sqrt{s}=546$ and $1800 \mathrm{GeV}$ yield $\alpha^{\prime}=0.34 \pm 0.07$. A fit including also the ISR data yields $\alpha^{\prime}=0.26 \pm 0.02$.

\section{Inelastic Non-Diffractive cross section}

The total inelastic rate has been obtained by measuring separately its diffractive and non-diffractive components. Inelastic non-diffractive events were contributed by the West East trigger coincidence. This trigger detected
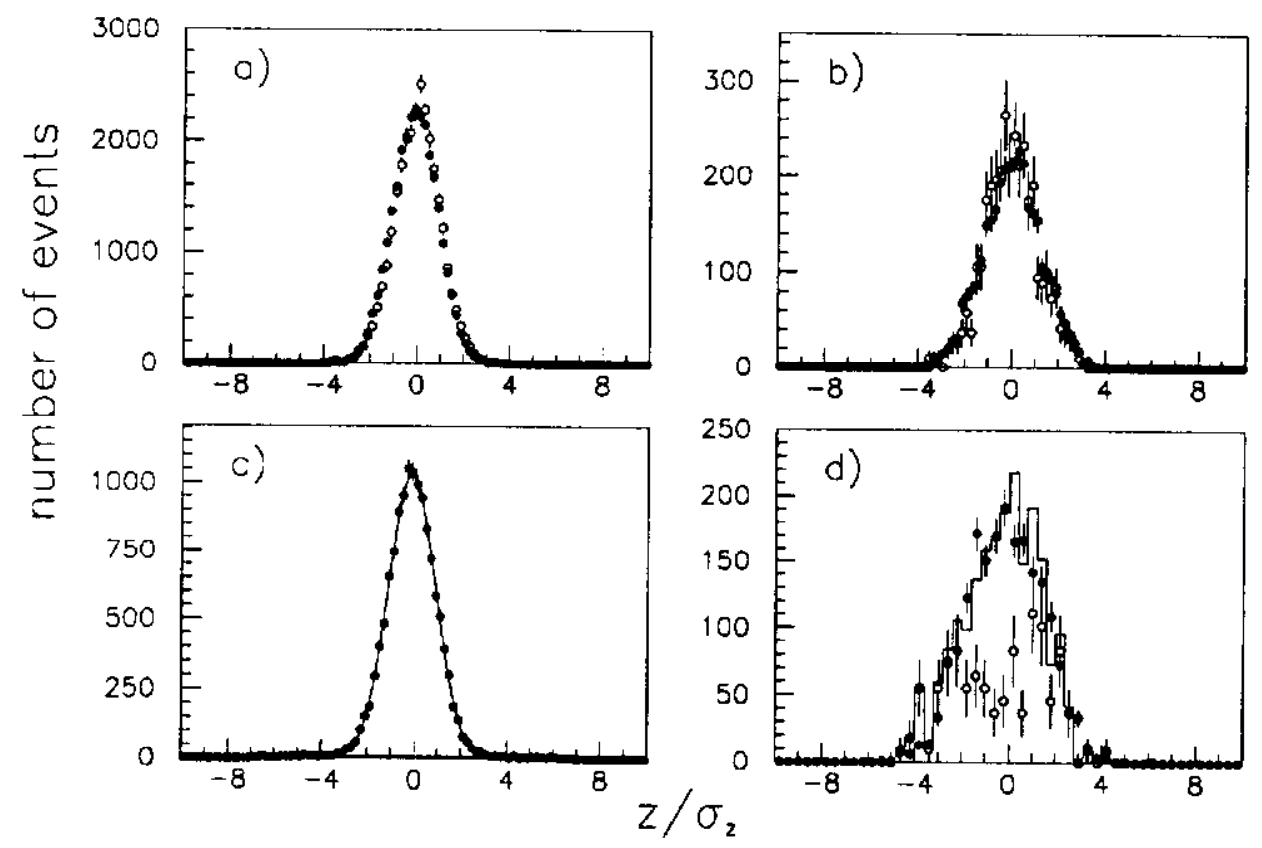

Figure 2: Vertex z-distributions in units of the spread $\sigma_{z}$. Solid points $(\bullet)$ are data. (a), (c): VTPC. (b),(d): forward telescopes.

(a),(b) $\sqrt{s}=546 \mathrm{GeV}$ : empty points (o) are simulated inelastic events reconstructed as the data and normalized to the number of measured events.

(c),(d) $\sqrt{s}=1800 \mathrm{GeV}$ : the solid line is the fit result. In (d) the empty points

(o) indicate the shape of tagged background events.

more than $98 \%$ of the inelastic non-diffractive events and only the high-mass tail of the single diffaction dissociation, for a total of 45770 (229325) events 
at $\sqrt{s}=546(1800) \mathrm{GeV}$. Most of the background triggers due to beam halo and/or beam-gas interactions were removed with a time of flight analysis of the trigger counters and by using the CDF vertex chamber (VTPC) pattern reconstruction capability to reject particle showers originating upstream of the interaction region. In the remaining events, all tracks were reconstructed in the pseudorapidity range $|\eta| \leq 6.7$. The tracks and the trigger counter timing information were combined to obtain the $z$-distribution of the event vertices. This distribution was used to estimate the residual background contamination by comparing it with Monte Carlo simulation. The input angular and multiplicity distributions for the simulation have been derived by existing data measured at $\sqrt{s}=200$ to $900 \mathrm{GeV}$ by the UA5 experiment [4]. The comparison showed no background at $\sqrt{s}=546 \mathrm{GeV}$, and a small contamination at $1800 \mathrm{GeV}$, where the event $z$-distribution was then fitted with the sum of the simulated distribution and of the background distribution measured on tagged background events, as shown in Fig. 2. At $\sqrt{s}=546(1800) \mathrm{GeV}, 31582 \pm 178$ $(13903 \pm 151)$ events were estimated to be real $\bar{p} p$ interactions. The simulation was also used to estimate the corrections for events without a valid vertex $(<0.5 \%)$ or lost due to the partial angular coverage of the trigger $(\simeq 1 \%)$. The number of inelastic events was finally multiplied by the trigger prescaling factors before being used in the cross section calculation.

\section{Single Diffractive Dissociation}

Single diffractive events are characterized by the presence of a quasi-elastic recoil antiproton with a differential distribution sharply peaked at both small angles and small diffracted masses $M_{x} \approx \sqrt{s \cdot(1-x)}$ with $x=p_{\bar{p}} / p_{\text {beam }}$. These events were contributed by the trigger $\bar{p} \cdot$ East, which required the coincidence of proton fragments on the East side of the interaction region and of an antiproton detected on the West side of the small angle spectrometer, where a string of Tevatron dipoles allowed to measure the $\vec{p}$ momentum with a resolution $\sigma_{p} / p \simeq 0.1 \%$. This good resolution allowed to separate the single diffraction dissociation from the non-diffractive contribution in the $\vec{p}$ momentum distribution. From 15272 (73480) triggers at $\sqrt{s}=546(1800) \mathrm{GeV}$ we selected good interactions as in the elastic and inelastic non-diffractive triggers, by requiring the validation of both the antiproton trajectory and the event vertex. The background contamination ( $\ll 1 \%$ at $\sqrt{s}=546 \mathrm{GeV}, \sim 1 \%$ at $1800 \mathrm{GeV}$ ) was statistically subtracted from the recoil $d^{2} N / d M_{x}^{2} d t$ distribution. Finally, events were restricted to the $\left(M_{x}^{2}, t\right)$-region in which the geometrical spectrometer acceptance was larger than 0.02 . The final sample contained 4604 (3671) 
events at $\sqrt{s}=546(1800) \mathrm{GeV}$.

The single diffraction dissociation contribution was estimated by fitting the $d^{2} N / d M_{x}^{2} d t$ distribution of the data with the standard triple-Pomeron Regge formula $s d^{2} \sigma_{s d} / d M_{x}^{2} d t=G(t)\left(s / s_{0}\right)^{\alpha_{p}(0)-1}\left(s / M_{x}^{2}\right)^{2 \alpha_{p}(t)-\alpha_{p}(0)}$. Following tradition a linear Pomeron trajectory was assumed: $\alpha_{P}(t)=1+\epsilon+\alpha^{\prime} t$. The form used for the fit is thus $d^{2} \sigma_{s d} / d M_{x}^{2} d t=G(0) \cdot\left(s / s_{0}\right)^{\epsilon} \cdot e^{\left(b_{0}+2 \alpha^{\prime} \ln \left(s / M_{x}^{2}\right)\right) t}$. $\left(s / M_{x}^{2}\right)^{1+\epsilon}$. In order to account for the non-diffractive contribution at $x \geq 0.85$, the empirical term $d^{2} \sigma_{n d} / d x d t=I \cdot(1-x)^{\gamma} \cdot e^{b^{\prime} t}$ was added to this formula. The sum of the two forms, corrected for the efficiency of detecting the products of a diffractive mass $M_{x}$ and for the $\bar{p}$-spectrometer acceptance and resolution, was fitted to the data with a maximum likelihood method. The total single diffractive rate was obtained by integrating the fitted distribution over the full momentum transfer in the diffraction region $1.5 \mathrm{GeV}^{2}<M_{x}^{2}<0.15 s$, and multiplying by the trigger prescaling factor and by a factor of two to account for the dissociation of the antiproton. The single diffraction contribution to the total inelastic rate, $R_{i n}$, was integrated after removing the events which also gave a West . East trigger. In the fit we assumed $\alpha^{\prime}=0.25 \mathrm{GeV}^{-2}$ in order to reduce the number of free parameters. Fit results are listed in Table 1. Data and fits are compared in Fig. 3.

Table 1: Fit results

\begin{tabular}{lcc}
\hline \hline & $\sqrt{s}=546 \mathrm{GeV}$ & $\sqrt{s}=1800 \mathrm{GeV}$ \\
\hline$\sigma_{s d}(\mathrm{mb})$ & $7.89 \pm 0.33$ & $9.46 \pm 0.44$ \\
$G(0) \cdot\left(s / s_{0}\right)^{\epsilon}\left(\mathrm{mb} / \mathrm{GeV}^{2}\right)$ & $3.55 \pm 0.35$ & $2.53 \pm 0.43$ \\
$\epsilon$ & $0.121 \pm 0.011$ & $0.129 \pm 0.022$ \\
$b_{0}\left(\mathrm{GeV}^{-2}\right)$ & $7.7 \pm 0.6$ & $4.2 \pm 0.5$ \\
$\sigma_{n d}(\mathrm{mb})$ & $1.2 \pm 0.2$ & $2.5 \pm 0.5$ \\
$I\left(\mathrm{mb} / \mathrm{GeV}^{2}\right)$ & $537_{-280}^{+498}$ & $162_{-85}^{+160}$ \\
$b^{\prime}\left(\mathrm{GeV}^{-2}\right)$ & $10.2 \pm 1.5$ & $7.3 \pm 1.0$ \\
$\gamma$ & $0.71 \pm 0.22$ & $0.10 \pm 0.16$ \\
\hline
\end{tabular}

The standard Regge form of the diffractive slope $b_{s d}=b_{0}+0.5 \mathrm{GeV}^{-2}$. $\ln \left(s / M_{x}^{2}\right)$ fits well our data at each energy. However, our fits yielded $b_{0}=$ $7.7 \pm 0.6 \mathrm{GeV}^{-2}$ at $\sqrt{s}=546$ and $b_{0}=4.2 \pm 0.5 \mathrm{GeV}^{-2}$ at $\sqrt{s}=1800$. This difference in the $b_{0}$ values could be a consequence of the fact that low masses $\left(M_{x}^{2} \leq 6 \mathrm{GeV}^{2}\right)$, which represent a large fraction of the resolution-dominated diffractive peak at $x \simeq 1$, have steeper slopes than those given by such formula. 

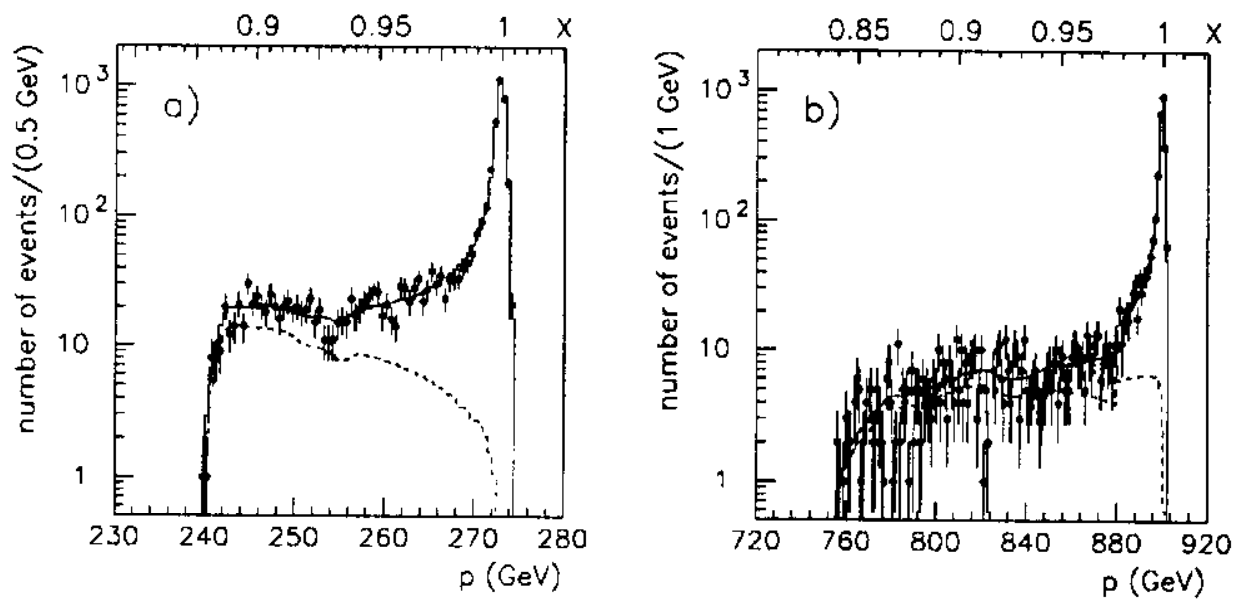

Figure 3: Recoil momentum distribution for all events at (a) $\sqrt{s}=546$ and (b) $\sqrt{s}=1800 \mathrm{GeV}$. The data $(\bullet)$ are not corrected for the spectrometer acceptance; the solid line represents our fit; the dashed line is the non-diffractive contribution

The parameter $\epsilon$ measures the deviation of the differential cross section $d \sigma_{s d} / d M_{x}^{2}$ from $1 / M_{x}^{2}$ dependence. Theoretically, $1+\epsilon$ can be interpreted as the intercept of a supercritical Pomeron, assuming that only the triple-Pomeron diagram contributes to diffraction dissociation and neglecting screening effects. The possible contribution of a PPR term, which has an $M_{x}^{-3}$ behaviour, cannot be resolved by fitting the mass distribution since this term is all contained in the resolution-dominated peak. However an additional PPR term would increase the low masses cross section. By studying the angular and multiplicity distribution of the dissociation products of these masses, a $1 \sigma$ bound of $15 \%$ is put on the PPR contribution to $\sigma_{s d}$. A corresponding systematical error $\delta \epsilon=-0.011$ is derived. By taking into account also a possible $\pm 0.1 \mathrm{GeV}^{-2}$ uncertainty on $\alpha^{\prime}$, final systematic errors on $\epsilon$ and $b_{0}$ are obtained: $\delta \epsilon= \pm 0.011$ and $\delta b_{0}=\mp 1.5 \mathrm{GeV}^{-2}$ are both energies. The overall systematical error on $\sigma_{s d}$ due to uncertainties on $\epsilon$ and on $\alpha^{\prime}$ is $<1 \%$.

\section{Conclusion}

Combining the elastic and inelastic rates, we obtain $\left(1+\rho^{2}\right) \cdot \sigma_{T}=62.64 \pm 0.95$ and $81.83 \pm 2.29 \mathrm{mb}$ at $\sqrt{s}=546$ and $1800 \mathrm{GeV}$, respectively. Assuming $\rho=0.15$, our results for the total cross section are $61.26 \pm 0.93 \mathrm{mb}$ at $\sqrt{s}=546$ and $80.03 \pm 2.24 \mathrm{mb}$ at $\sqrt{s}=1800 \mathrm{GeV}$. 
The elastic scattering cross sections are $12.87 \pm 0.30(19.70 \pm 0.85) \mathrm{mb}$ at $\sqrt{s}=546$ (1800) $\mathrm{GeV}$. From the elastic and total cross section values we derive the ratio $r=\sigma_{e l} / \sigma_{T}=0.210 \pm 0.002(0.246 \pm 0.004)$.

The continuing rise of $r$ up to $\sqrt{s}=1800 \mathrm{GeV}$ is in qualitative agreement with the basic hypothesis of various optical models in which the nucleon opacity increases with $s$, but the present energy is still far below the asymptotic regime of black-disk maximum absorption at which $r=0.5$. However, the central opaqueness of the nucleon, defined as $\operatorname{Im} f(s, b)$ at $b=0$, where $f(s, b)$ is the elastic scattering amplitude in terms of the impact parameter $b$, has increased from 0.36 at the ISR to $0.492 \pm 0.008$ at $\sqrt{s}=1800 \mathrm{GeV}$ and is close to the unitarity bound of 0.5 corresponding to complete absorption.

The total inelastic cross sections are measured to be $48.39 \pm 0.66(60.33 \pm 1.40)$ $\mathrm{mb}$ at $\sqrt{s}=546(1800) \mathrm{GeV}$. Single diffraction dissociation contributes with $7.89 \pm 0.33(9.46 \pm 0.44) \mathrm{mb}$.

In terms of single-Pomeron exchange, the $\bar{p} p$ total cross section, $\sigma_{T}$, behaves at high energies as $s^{\alpha_{p}(0)-1}=s^{\epsilon}$. From our measurements of $\sigma_{T}$ at $\sqrt{s}=546$ and $\sqrt{s}=1800$ we derive $\epsilon=0.112 \pm 0.013$. This $\epsilon$-value is in good agreement with the average $\epsilon$-value $0.125 \pm 0.010 \pm 0.011$ syst. obtained from the single diffraction $M_{x}^{2}$-dependence at our two energies. In terms of triplePomeron exchange, when using $\epsilon=0.112 \pm 0.013$ (from the rise of $\sigma_{T}$ ) and $b_{0}=6.0 \mathrm{GeV}^{-2}$ (average over our two energies), the value of $\sigma_{s d}=7.89 \pm 0.33$ $\mathrm{mb}$ measured at $\sqrt{s}=546$ extrapolates to $\sigma_{s d}=13.9 \pm 0.9$ at $\sqrt{s}=1800$, where we measure $\sigma_{s d}=9.46 \pm 0.44 \mathrm{mb}$. This clearly indicates that large screening corrections have to be introduced in order to save the traditional supercritical Pomeron model.

\section{References}

[1] F.Abe et al. Nucl. Instrum. Methods Phys. Res., Sect. A 271 (1988) 387. [2] F.Abe et al. FERMILAB-PUB-93/232-E, FERMILAB-PUB-93/233-E, FERMILAB-PUB-93/234-E. Submitted to Phys. Rev. D.

[3] M.Bozzo et al. Phys. Lett. 147B, (1984) 385. G.Matthiae in Results and Perspectives in Particle Physics, La Thuile (1993), edited by M.Greco (Edition Frontieres, 1993).

[4] G.J.Alner et al. Zeit. fuer Phys. C33, (1986) 1. G.J.Alner et al. Phys. Rep. 154C, (1987) 247. 